\begin{tabular}{|l|c|c|c|}
\hline Eiszeitalter u. Gegenwart & 30 & $\begin{array}{c}213-220 \\
5 \mathrm{Abb} .\end{array}$ & Hannover 1980 \\
\hline
\end{tabular}

\title{
Transgression und Umlagerung im Gebiet des Helgoland-Riffs
}

\author{
FRIEDRICH WUNDERLICH *) \\ Transgression, redeposition, core cuttings, abraison, bar, Pleistocene \\ North Sea (Helgoland-Riff Area).
}

\begin{abstract}
Kurzfassung: Im Gebiet des Helgoland-Riffs (pleistozänes Reliktmaterial) wurden Längs- und Querprofile mit Sedimentechograph und Side-Scan gefahren. Die Profile wurden abgegriffen, abgebohrt und abgedredgt.

Einer meist nur geringmächtigen rezenten Deckschicht sind mit einer häufig scharf ausgeprägten Erosionsdiskordanz subrezente und fossile Sedimente untergelagert. Es wurden marine Fazies, Watten- und Brackwasserfazies sowie Torfe, Schmelzwassersande und Pleistozän (Geschiebematerial) angetroffen.

Das Untersuchungsgebiet unterliegt z. Z. noch der Erosion. Bei Sturm wird stark umgelagert, grober Sand ist in vielen Fällen wohl als "residual concentration" anzusehen. Aber auch Schlicklagen von mehr als $10 \mathrm{~cm}$ Mächtigkeit werden rezent in die Oberflächenschichten eingeschaltet.
\end{abstract}

\section{[Transgression and Redeposition in the Helgoland-Riff Area]}

A bstract: In the Helgoland-Riff area (Pleistocene relict material), profiles were investigated by sediment echo sounder and side-scan sonar. Samples were taken on the profile lines by grab sampler, dredges and vibro-coring.

A usually thin cover of recent deposits overlies subrecent and fossil sediments with an often sharp, erosion discontinuity plane. Sediments of marine facies, tidal flat and brackish facies, as well as peat, Pleistocene melt water sand and morainic material were found.

At present, the investigated area is still being eroded. Sediment transport takes place under storm conditions, and coarse sand has to be looked upon in many cases as lag deposit. But mud layers more than $10 \mathrm{~cm}$ thick also occur intercalated in the recent surface layers.

\section{Einführung}

Das Helgoland-Riff gehört zu einer durch pleistozäne Ablagerungen entstandenen Untiefenzone, die sich nach NNW über das Sylt-Außenriff und die Turbo-Bank bis zum Kleinen Fischer-Riff, etwa 80 sm westlich des Limfjords, erstreckt (Abb. 1).

Bereits im Eem wurde dieses Gebiet einmal vom Meer zurückerobert, da während der letzten Vereisung die skandinavischen Gletscher dieses Gebiet nicht mehr erreichten. Fossile und rezente Transgressionen haben demnach zu seiner Gestaltung ebenso beigetragen wie subaerische dynamische Vorgänge während der Weichselvereisung. Die letzte Transgression fiel in eine Zeit sehr raschen Meeresspiegelanstieges (BEHRE \& MENKE 1969; SiNDOWSKi \& Streif 1974; Kolp 1976; Menke 1976; Streif 1978). Das bedeutet, daß der Überflutungsvorgang bereits etwa 7500 v.h. abgeschlossen war, wenn auch nicht auszuschließen ist, daß einige Untiefen noch etwas länger exponiert waren.

*) Anschrift des Verfassers: Dr. F. W u n d e r li ch, Institut für Meeresgeologie und Meeresbiologie „Senckenberg", Schleusenstraße 39 A, D-2940 Wilhelmshaven. 


\section{Fragestellung, Arbeitstechniken}

In den Jahren 1977 und 1978 wurde das Gebiet des Helgoland-Riffs auf den Transgressionskontakt hin untersucht. Ferner sollte Fragen der Aufarbeitung und Verteilung der Sedimente nachgegangen werden (WUNDERLICH 1979).

Hierbei wurde folgendermaßen vorgegangen: zunächst wurden mit dem Sedimentechograph ELAC LAZ 71 CA 908 Längs- und Querprofile gefahren, synchron dazu erfolgte die flächenhafte Aufnahme mit Side-Scan. Anhand der Aufzeichnungen wurden

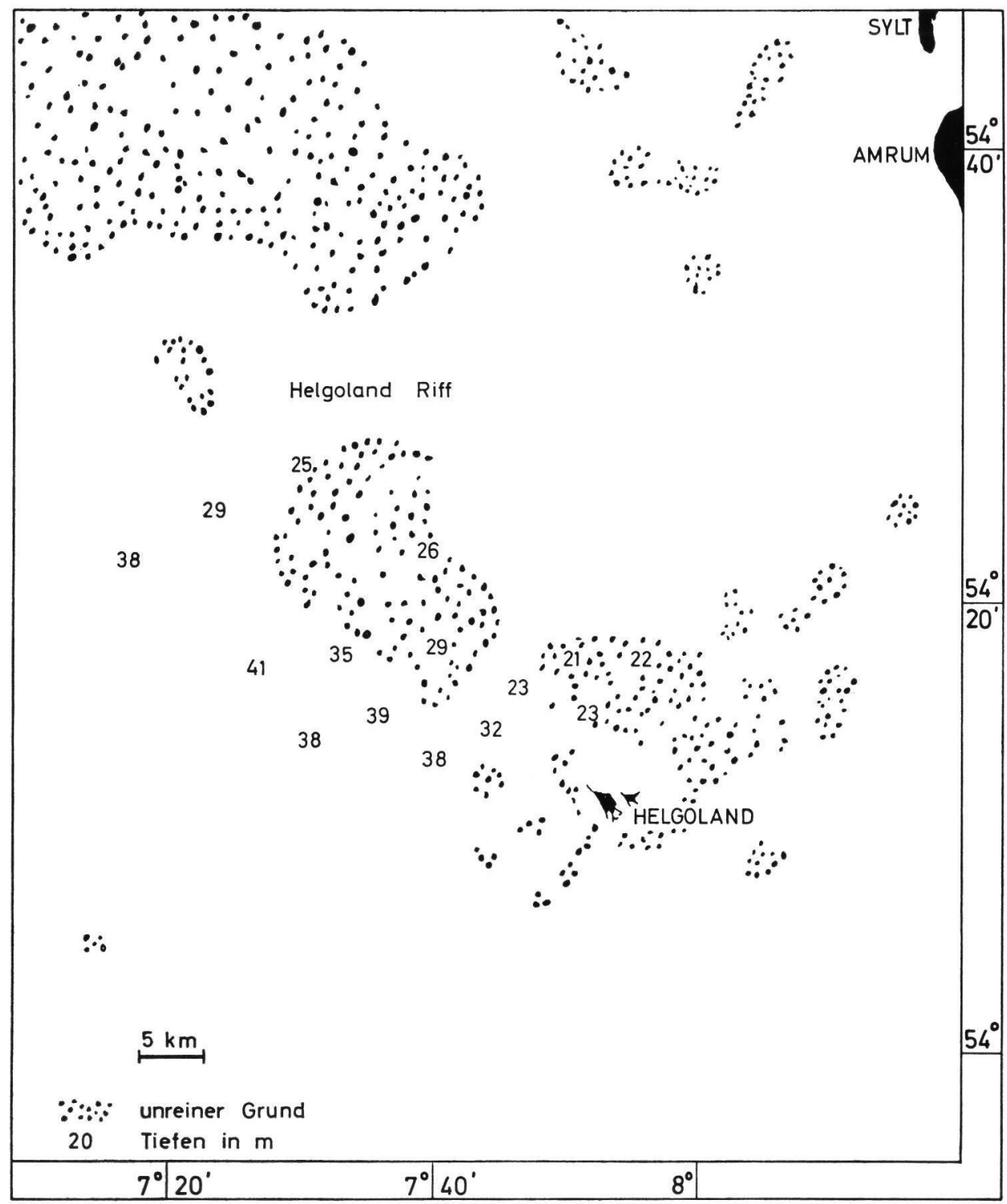

Abb. 1: Lage des Helgoland-Riffs. 
dann die Stationspunkte bestimmt. Zum Einsatz kamen der Vibrohammer nach KöGLER (Kögler et al. 1972) sowie an gleicher Position der Kastengreifer nach REINEck (REINECK 1963). Der Kastengreifer wurde vor allem deshalb gleichzeitig eingesetzt, um ein besseres Bild der oberflächennahen Strukturen zu erhalten, die beim Vibrohammer erfahrungsgemäß meist in Mitleidenschaft gezogen werden. Ebenso ist dadurch mehr Material für Sedimentanalysen vorhanden.

Abschnittweise wurden die Profile mit der Hartgrunddredge abgesammelt, die Verteilung und Zonierung von Schill, Steinen und oberflächennaher Fauna ließ sich damit brauchbar kartieren.

\section{Auswertung}

Die Auswertung der Proben anhand von Gefüge, Korngrößen, Mikro- und Makrofauna sowie groben Bestandteilen ist noch nicht abgeschlossen, auch sind die Profile noch nicht vollständig abgebohrt. Erste Ergebnisse zeigen das folgende Bild.

Morphologie, Innen-Großgefüge, Sedimentdecke, A uf arbeitung: Bereits die Echographenaufnahmen ließen sehr oft einen mit der rezenten, unausgeglichenen Oberflächenmorphologie in keinem Zusammenhang stehenden Innenaufbau erkennen. In verschiedene Richtungen einfallende Schrägschichten, wannenartig ausgefüllte Senken und Schichten unterschiedlicher Schallimpedanz ließen eine uneinheitliche Zusammensetzung erkennen. Am Westrand des Helgoland-Riffs wurde eine kliffartige und später offensichtlich aufgefüllte Struktur angetroffen (Abb. 2). Erosion findet an der W-Seite heute offenbar auch in tieferen Bereichen statt: ältere, horizontal abgelagerte Schichten streichen gegen den Hang hin aus.

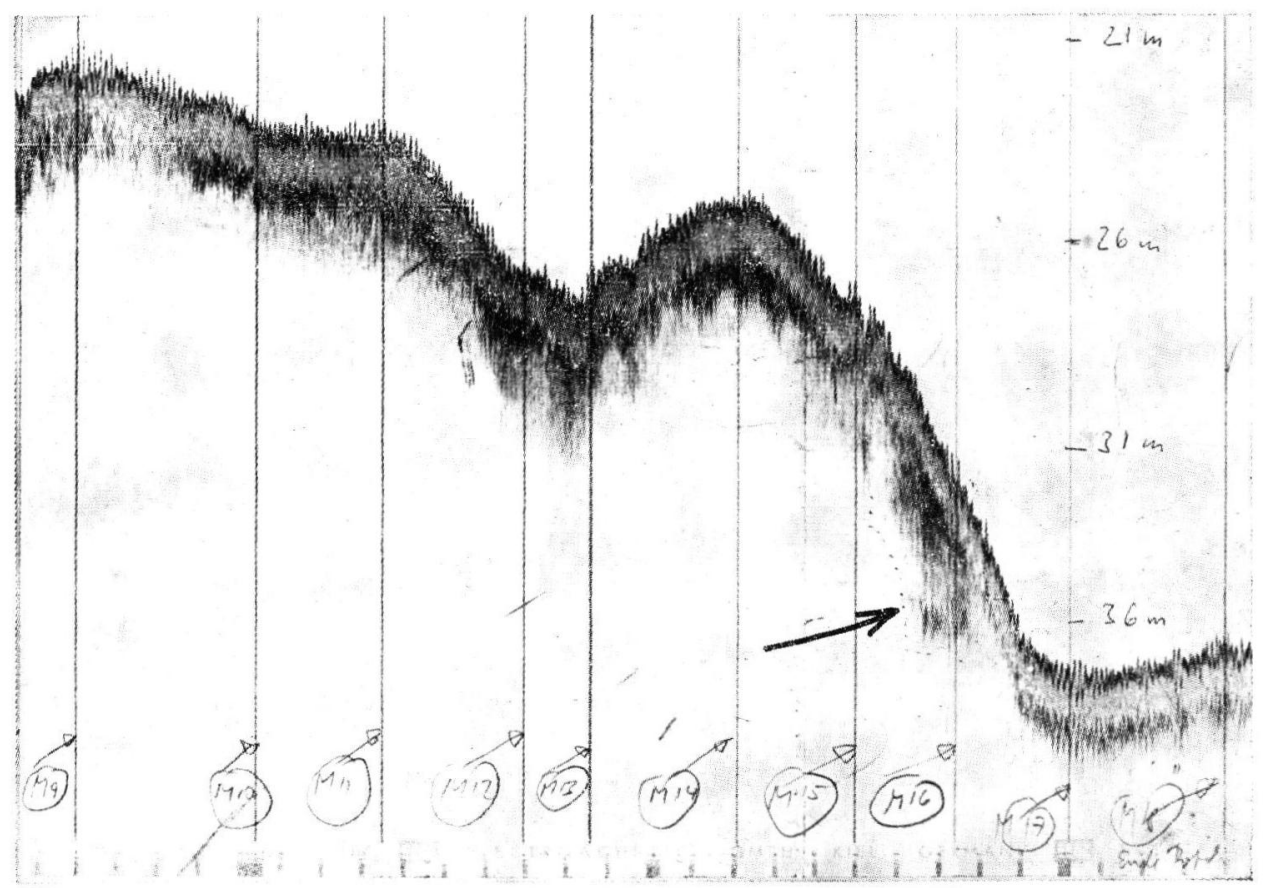

Abb. 2: An der W-Seite des Helgoland-Riffs wurde innerhalb eines aufgefüllten Kliffs (Pfeil) Brackwasserfauna gefunden. 
Die rezente Sedimentdecke ist i. a. recht geringmächtig, meist wenig mehr als $50 \mathrm{~cm}$. Gelegentlich werden auch aufgefüllte Kolke gefunden. Nach E zu stehen aufgearbeitete Kiese und Blöcke direkt an (DeccA r.21.32/p. 70.28). Die Sedimentoberfläche zeigt keine einheitliche Zusammensetzung. Zwar herrschen in der Regel gröbere, helle Sande vor, doch wird auch Schlick angetroffen (DECCA r.11.96/p. 77.60, dunkle, schlickige Sedimente über gut sortiertem Kies). Wenn natürlich auch mit älterem, freigelegten Sediment gerechnet werden muß, so sind doch im rezenten Schichtverband eingeschaltete Schlicklagen von mehr als $10 \mathrm{~cm}$ Mächtigkeit zu finden. Später freigelegt, geben sie durch ihre Kompaktion gewissen Schutz gegen Erosion (Abb. 3).

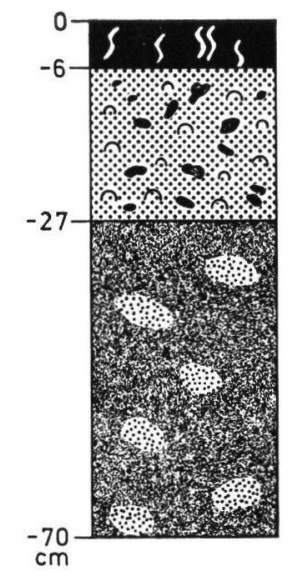

Abb. 3: Kern mit rezentem, mit Gängen durchsetztem Schlick $(0-6 \mathrm{~cm})$, marinem Sand mit Schill und Schlickgeröllen/Schlickflasern $(-6$ bis $-27 \mathrm{~cm})$ und schlickig-bindigem Sediment mit sandigen Nestern $(-27$ bis $-70 \mathrm{~cm})$.

Meist ist das Sediment dicht mit Herzseeigeln, Scheidenmuscheln und vor allem am W-Rand mit Islandmuscheln besiedelt. Doch zeigen gut erhaltene Schrägschichtungen, laminierte Sande und Fluchtspuren des Herzseeigels (Wunderlich 1979) starke Umlagerungen an. Bei Wassertiefen zwischen -25 und $-30 \mathrm{~m}$ ist das vor allem auf Seegang bei Sturm aus westlichen Sektoren zurückzuführen.

Stürme aus östlichen Sektoren können nicht so stark auf den Untergrund einwirken, der fetch ist hier erheblich geringer, die Wassertiefen nehmen nach $\mathrm{E} \mathrm{zu} a b$. Damit ist wohl auch der Transport von klastischem Material aus dem Helgoland-Riff nach W nur gering. Kontrollkerne aus der schlickigen Senke westlich des Hanges zeigten lediglich große A Ahnlichkeit mit den Schlickgebieten südlich Helgoland hinsichtlich Fauna, Verwühlung und Korngröße.

Der Transport klastischer Komponenten innerhalb des Helgoland-Riffs scheint nicht allzu groß zu sein, wie vor allem die Gefügebilder und Korngrößen in den Kastengreiferproben zeigen. Auch die oben erwähnten bis zu $20 \mathrm{~cm}$ (!) mächtigen Schlicklagen innerhalb der rezenten Residualsande können als Indikator hierfür gelten: die Mikrofauna ergab ein heutzeitliches Spektrum. Anerodierte, ältere Schlicke scheiden also hier aus.

Das wirft die Frage auf, inwieweit Schlick resuspendiert und wieder abgelagert werden kann. Falls es sich nicht um Material handelt, welches bei Sturmfluten aus dem Gebiet der nordfriesischen Westküste kommt (GADOw \& REINECK 1969), muß es im Bereich 
des Helgoland-Riffs in Suspension geraten sein. Möglich wäre auch die Befrachtung mit aufgearbeitetem Schlick von der W-Kante des Riffs, die z. Z. ja noch unter Erosion steht und vor allem bei Sturm aus westlichen Sektoren Materialverluste erleidet, während sie bei östlichen Winden möglicherweise etwas als Sedimentfalle gelten kann. Auf jeden Fall müssen lokal beträchtliche Gehalte an Tonmineralien im Wasser enthalten sein, die auch rasch wieder abgelagert werden können (MCCAvE 1971). Hierbei ist sicher auch das unruhige, morphologische Relief von Bedeutung.

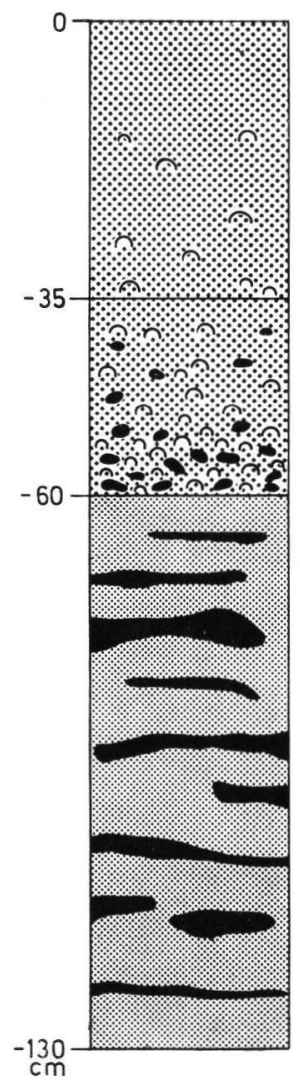

Abb. 4: Kern aus dem Kliff am W-Hang des Helgoland-Riffs (siehe: Abb. 2). Unterhalb einer $60 \mathrm{~cm}$ mächtigen, rezenten Deckschicht wird eine brackische Wechselschichtung angetroffen.

Kontaktzone rezent/fossil; ältere Sedimente. Die Grenzlinie rezent/subrezent/fossil ist im allgemeinen als scharf ausgebildete Erosionsdiskordanz mit einem Basiskonglomerat versehen (Abb. 3, Abb. 4, Abb. 5). Die Fauna dringt dabei nur selten in die älteren, unterlagernden Schichten ein. Auch dies ist ein Hinweis auf die insgesamt noch erosive Situation des Helgoland-Riffs. Vergleichbar ist die Situation vor Sylt, wo ja ebenfalls meist dicht unterhalb der rezenten Sedimentdecke ältere Schichten anstehen. 


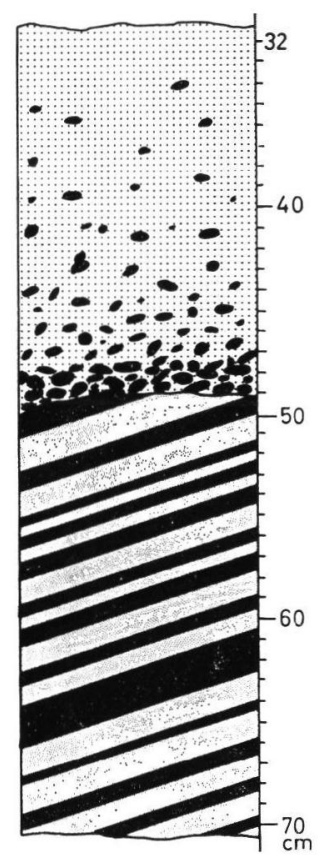

Abb. 5: Ausschnitt aus einem Kern. Unter dem Basiskonglomerat schräg einfallende, nichtmarine Wechselschichten aus grauweißem Sand und Torfgrus. Scharfe Erosionsdiskordanz.

(Nach WunderLich 1979).

Schwieriger sind Lokalitäten zu deuten, wo sich marin/marin verzahnt, wobei die unteren Schichten auch subrezent oder fossil sein können. Auf Pos. Decca r.22.92/p. 78.78 wurde unter einer $65 \mathrm{~cm}$ mächtigen, einheitlich sandig-marinen Deckschicht mit einer scharfen Grenze feinerer Sand angetroffen, der von Seeigeln verwühlt war, aber nicht rezent entstanden zu sein schien. Mit weiteren scharfen Grenzlinien fanden sich darunter laminierte Sande, Wechsellagen von Sand und Ton und bioturbate Gefüge, wahrscheinlich durch Polychaeten erzeugt.

Wenn auch die Überflutung des Gebietes zwischen 9000 und 7500 v.h. geologisch gesehen recht rasch erfolgte, war bei dem unausgeglichenen Relief zwischenzeitlich die Ausbildung von Watten möglich. Auf Pos. Decca r.8.18/p. 73.78 lagen unter einer etwa $42 \mathrm{~cm}$ mächtigen Deckschicht von braunen Sanden mit Steinchen (Kies) und Schill nach einem kurzen Übergang (Aufarbeitungszone) graue Sande mit Cerastoderma edule, also einer Wattform. Dagegen wurden auf Pos. DeccA r.18.54/p. 67.92 keine scharfen Grenzen innerhalb eines Kernes angetroffen. Unterhalb $25 \mathrm{~cm}$ verwühltem, schlickigem Sediment fanden sich bis $-170 \mathrm{~cm}$ schlickige Feinsande mit viel Schill und Bruchschill, stark bioturbat und in den tieferen Teilen ebenfalls mit Klappen von Cerastoderma edule. Faunistisch gesehen also ebenfalls Watt. Wenn man auch nicht die Möglichkeit ausschließen kann, daß ältere Sedimente in Sedimentfallen zusammengespült wurden, ist dennoch das ehemalige Vorkommen von Watten augenfällig.

Noch aufschlußreicher ist Pos. Decca r.17.72/p. 66.12 (Abb. 2, Abb. 4). Hier wurde das oben erwähnte Kliff angebohrt. Auf den Transgressionskontakt, der bei $-60 \mathrm{~cm}$ lag, 
folgten sandig/tonige, teils stark verwühlte Wechselschichten mit Jadammina polystoma, Elphidium gunteri und Rotaria beccarii, also eine ausgeprägte Brackwasserfauna (für die Bestimmung der Mikrofauna bin ich Dr. G. Richter zu Dank verpflichtet). Natürlich muß auch damit gerechnet werden, daß eemzeitliche Ablagerungen angetroffen werden. Wahrscheinlicher aber ist diese Brackwasserfauna dem holozänen Meeresspiegelanstieg zuzuordnen. Erosion durch westliche Stürme kann seither beträchtliche Schichtverluste an der W-Kante hervorgerufen haben.

Torf wurde ebenfalls im Untergrund anstehend angetroffen (DECCA r.19.26/p. 74.44). Häufig findet man auch schwarzen Torfgrus in dicken Lagen als Wechselschichtung mit grauen, nichtmarinen Sanden (Abb. 5), Bernstein liegt darin ebenfalls vor. Die Vermoorung während des Eem oder Holozän scheint also recht ausgeprägt gewesen zu sein.

\section{Schriftenverzeichnis}

Behre, K.-E. \& Menke, B. (1969): Pollenanalytische Untersuchungen an einem Bohrkern der südlichen Doggerbank. - Dtsch. Akad. Wiss. Berlin, Beitr. Meereskde., 24/25: 122-129; Berlin.

Gadow, S. \& Reineck, H.-E. (1969): Ablandiger Sandtransport bei Sturmfluten. - Senckenbergiana maritima, (1) 50: 63-68. 5 Abb., 1 Tab., 1 Taf.; Frankfurt a. M.

Kögler, F.-C. Seibold, E. \& Veit, K. H. (1972): Die Entnahme von Sandkernen aus der Brandungszone mit Vibrohammerkerngerät "Kiel-3“ und Hubschraubereinsatz. - Ber. Komm. Eur. Gemsch., Büro Eurisotop, 75: 1-34, Abb. 1-8, 1 Tab., Taf. 1-6; Brüssel.

KolP, O. (1976): Submarine Uferterrassen der südlichen Ost- und Nordsee als Marken des holozänen Meeresspiegelanstiegs und der Überflutungsphasen der Ostsee. - Petermanns Geogr. Mitt., $120: 1-23$; Gotha/Leipzig.

McCave, I. N. (1971): Wave effectivness at the sea bed and its relationship to bed forms and deposition of mud. - J. sediment. Petrol., 41: 89.96, Abb. 1-7; Tulsa, Okla.

Menke, B. (1976): Befunde und Uberlegungen zum nacheiszeitlichen Meeresspiegelanstieg (Dithmarschen und Eiderstedt, Schleswig-Holstein). - Probl. Küstenforsch., 11: 145-161; Hildesheim.

Sindowski, K.-H. \& Streif, H. (1974): Die Geschichte der Nordsee am Ende der letzten Eiszeit und im Holozän. - In: Woldstedt, P. \& Duphorn, K. (Hrsg.): Norddeutschland und angrenzende Gebiete im Eiszeitalter, 411-431. - Stuttgart (Köhler).

Streif, H. (1978): Geologie des Untergrundes. 1. Geologie des Nordseebeckens. 2. Geologie des Küstenraumes. 3. Der holozäne Meeresspiegelanstieg und die heutige Küstengestalt. - In: ReineCK, H.-E. (Hrsg.): Das Watt, 19-38; Frankfurt a. M.

Reineck, H.-E. (1963): Der Kastengreifer. - Natur u. Mus., 93: 5 Abb., 102-108; Frankfurt a. M.

Wunderlich, F. (1979): Sandauflagerung und Herzigel-Fluchtspuren im Helgoland-Riff. — Natur und Museum, 109: 315-319, Abb. 1-5; Frankfurt a. M.

Manuskript eingegangen am 1. 2. 1980. 
Friedrich Wunderlich 